\title{
Search for supersymmetry with compressed mass spectra or decays via Higgs bosons at CMS
}

\author{
Constantin Heidegger* \\ on behalf of the CMS Collaboration \\ ETH Zurich, Institute for Particle Physics and Astrophysics, \\ Otto-Stern-Weg 5, 8093 Zurich, Switzerland \\ E-mail: constantin.heidegger@cern.ch
}

In this talk, a review of searches for supersymmetric particles with very compressed mass spectra and searches for supersymmetric particles that decay via Higgs bosons is presented. All searches have used $35.9 \mathrm{fb}^{-1}$ of $13 \mathrm{TeV}$ data collected by the CMS detector at the CERN LHC in 2016.

EPS-HEP 2017, European Physical Society conference on High Energy Physics 5-12 July 2017

Venice, Italy

${ }^{*}$ Speaker. 


\section{Introduction}

Supersymmetry (SUSY) is an elegant extension to the standard model of particle physics (SM) which has proven to be incomplete. Proton-proton collision experiments such as CMS [1] make huge efforts in conducting a diverse program of searches for the production of new particles compatible with SUSY predictions in both colored and electroweak scenarios. In this talk, attention is firstly directed to SUSY models comprising compressed mass spectra. Thereafter, scenarios with decays via Higgs $(\mathrm{H})$ boson candidates are discussed.

\section{Searches in Compressed SUSY Spectra}

In the search for $R$-parity conserving SUSY, one expects to produce SUSY particles (or "sparticles") in pairs. Each of these SUSY particles then should decay via SM particles to a lightest SUSY particle (LSP), hence, one expects two LSPs in the final state. Depending on the mass difference or "mass splitting", $\Delta m$, between the produced sparticles and the LSPs, the SUSY particle spectrum either is called "compressed" (small $\Delta m$ ) or "non-compressed" (large $\Delta m$ ). The mass splitting is of relevance to the extent that it determines the possible decay products, and therefore the final state. Compressed spectra are experimentally challenging as the detectable SM particles have low transverse momentum $p_{\mathrm{T}}$ (they are "soft") - on the level of few GeV. Initial state radiation (ISR) is becoming increasingly relevant given that it boosts the sparticle system and produces large missing transverse energy $\left(p_{\mathrm{T}}^{\text {miss }}\right)$. Compressed models are interesting also from a theoretical point of view, e.g. their relic density is expected to be consistent with cosmological observations [2].

In this talk, compressed spectra in colored (mainly in scenarios of stop-pair production) and electroweak SUSY (chargino-neutralino production) is discussed.

\section{Stop-Pair Production}

Figure 2 shows the available decay modes for stop-pair production in a very compressed scenario where $\Delta m<m_{\mathrm{W}}$. This means, the decay cannot be mediated by an on-shell $\mathrm{W}$ boson but merely by an off-shell W boson (left diagram). Alternatively, the top superpartner can undergo a 4-body decay (middle diagram) or it can decay via flavor-changing neutral currents (FCNC) to a charm quark and the LSP (right diagram).


Figure 1: Simplified models of stop pair production decaying via $\widetilde{\chi}_{1}^{ \pm}$and off-shell $\mathrm{W}$ bosons (left), undergoing a 4-body decay (middle) or a decaying via flavor-changing neutral currents (right). Figures taken from [5]. 


\section{Searches for FCNC SUSY}

The FCNC scenario is probed by searching for events with two c-tagged jets and $p_{\mathrm{T}}^{\text {miss }}$ [3]. The discriminating power between signal and background arises from a combination of kinematic variables such as $p_{\mathrm{T}}^{\text {miss }}, H_{\mathrm{T}}, N_{\text {cjets }}$ which are used to build 23 search regions. Alternatively, one can probe FCNC SUSY by means of the $M_{\mathrm{T} 2}$ variable [4], which provides a great signal-to-multijet background discrimination. Stop-pair production with FCNC decays is excluded by these two analyses up to a stop mass of approximately $550 \mathrm{GeV}$.

\section{The Hadronic and Inclusive Stop-Pair Production Search}

The hadronic and inclusive stop-pair production search [5] aims to cover the full parameter space while exhibiting an optimized strategy in the compressed as well as in the non-compressed scenario. One expects at least two potentially b-tagged jets with $p_{\mathrm{T}}>20 \mathrm{GeV}$ and significant $H_{\mathrm{T}}$ due to ISR. However, as shown in Figure 2 (left), the smaller the $\Delta m$ the lower the $p_{\mathrm{T}}$ of the jets and hence the jets may not pass the $p_{\mathrm{T}}$ threshold. In the very compressed case, this can result in a substantial loss of signal efficiency. One therefore deploys a "soft b-tagging algorithm" in addition to the default b-tagged jets by looking at secondary vertices which do not have any jet but merely low- $p_{\mathrm{T}}$ tracks associated to it. In this way, the signal selection efficiency could be improved by $20 \%$. The 53 dedicated low- $\Delta m$ search regions provide good sensitivity for the models of compressed stop-pair production. Interpretations of the results for the chargino-mediated stop decay (middle) and the 4-body decay scenarios (right) are presented in Figure 2.


Figure 2: $p_{\mathrm{T}}$ spectrum of simulated $\mathrm{b}$ jets in signal models of stop-pair production for three different assumptions on $\Delta m$ (left), interpretation of the results in the chargino-mediated scenario (middle), and the 4-body decay scenario (right). Figures taken from [5].

\section{Search with one Soft Lepton}

One can search for compressed stop-pair production models also by requiring one soft lepton which then is expected to stem from the off-shell $\mathrm{W}$ boson decay [6]. Soft here means $p_{\mathrm{T}}$ as low as 5 (3.5) $\mathrm{GeV}$ in the case of e $(\mu)$. Significant contributions from ISR $\left(H_{\mathrm{T}}>300 \mathrm{GeV}\right)$ are necessary to generate large values of $p_{\mathrm{T}}^{\text {miss }}$ and to be able to discriminate between signal and background. Given that the $p_{\mathrm{T}}$ spectrum of the lepton depends on the mass splitting, the events are categorized 
in bins of the lepton- $p_{\mathrm{T}}$. The exclusion limits for chargino-mediated and 4-body stop decays is compatible with those obtained by the inclusive search.

\section{The Soft Opposite-Sign Dilepton Search}

A dedicated lepton identification technique and trigger strategy had to be developed in order to search for SUSY in events with two leptons of opposite charge and with $p_{\mathrm{T}}$ as low as 5 (3.5) GeV for $\mathrm{e}(\mu)$ [7]. In the double muon case, the $p_{\mathrm{T}}^{\text {miss }}$ at trigger level could be lowered to $125 \mathrm{GeV}$. While the reconstructed $M_{\tau \tau}$ mass can be used to discriminate against the dominant Drell-Yan background, one of the biggest challenges is to reject events with mis-reconstructed leptons. This is done by dedicated multi-variate analysis (MVA) techniques based on a boosted decision tree (BDT). The results can be interpreted in both stop-pair production (left) and the electroweak scenario (middle and right) as shown in Figure 3. The electroweak scenario here refers to $\widetilde{\chi}_{1}^{ \pm} \widetilde{\chi}_{2}^{0}$ production with decays via off-shell $\mathrm{W}$ and $\mathrm{Z}$ bosons.


Figure 3: Interpretation of the results of the search in events with two soft opposite-sign leptons in a stoppair production scenario (left), and the electroweak scenario (right). The electroweak scenario is $\widetilde{\chi}_{1}^{ \pm} \widetilde{\chi}_{2}^{0}$ production with leptonic off-shell $\mathrm{Z}$ and hadronic off-shell $\mathrm{W}$ boson decays, and its diagram is presented in the middle. Figures taken from [7].

\section{Electroweak Scenario - the "WZ Corridor"}

Exclusion limits on the electroweak scenario also have been imposed by the inclusive multilepton search [8], as shown in Figure 4 (left), which provides good sensitivity in a broad region of the parameter space. However, one observes a significant loss of sensitivity along the "WZ corridor" - a region of parameter space where $\Delta m \sim m_{\mathrm{Z}}$, hence, the electroweak SUSY signal has similar kinematics as the dominant background of the analysis, namely SM WZ. In order to amend this situation, the event categorization has been revised, now including three bins in $H_{\mathrm{T}}$ in order to capture any shape difference between signal and background that is due to ISR. While in the case of no or little ISR $\left(H_{\mathrm{T}}<100 \mathrm{GeV}\right)$ signal and background are kinematically the same along the WZ corridor, the signal is expected to appear at larger values of $p_{\mathrm{T}}^{\text {miss }}$ due to the presence of the LSPs in the case of ISR $\left(H_{\mathrm{T}}>200 \mathrm{GeV}\right)$. In this way, the sensitivity could be improved dramatically along the WZ corridor [9], as shown in Figure 4 (right). 


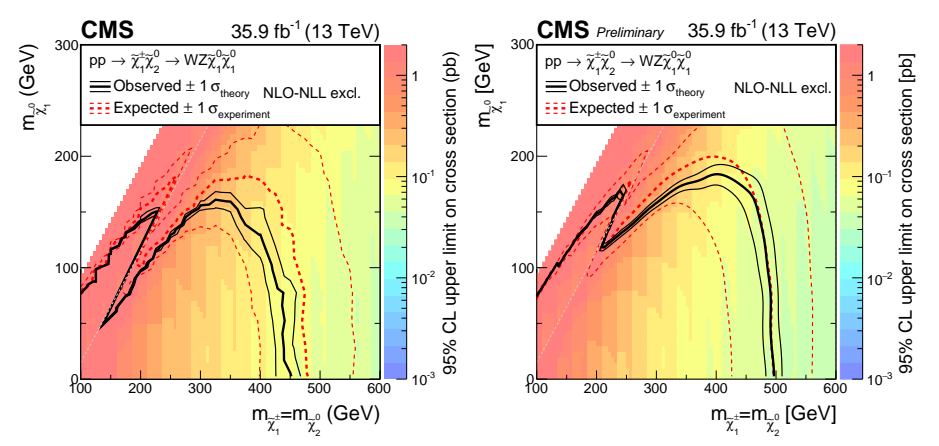

Figure 4: Interpretation of the results of the inclusive multilepton search [8] in the heavy slepton scenario (left), and interpretation of the results of the optimized WZ corridor analysis [9] in the same model (right). The sensitivity could be improved significantly along the WZ corridor. A small improvement is also visible for large values of $m_{\widetilde{\chi}_{1}^{ \pm}}=m_{\widetilde{\chi}_{2}^{0}}$ and $m_{\widetilde{\chi}_{1}^{0}} \sim 0 \mathrm{GeV}$.

\section{SUSY Searches with Higgs Bosons}

The mass eigenstates of SUSY particles that are produced via the electroweak interaction are mixtures of gaugino eigenstates. There are two ways of how a Higgs boson can occur in EWK SUSY processes: either in a WH-like signature [10] with a wino next-to-LSP and a bino LSP, or in a gauge-mediated SUSY breaking (GMSB) scenario with a higgsino next-to-LSP and a gravitino LSP. While extensive searches for these processes have been performed and reported recently by CMS [9], a search for a specific GMSB scenario is presented in the next section.

\section{Search for GMSB Scenario with 4 B's and Missing Transverse Energy}

The search reported in [11] is targeted at a GMSB scenario of higgsino $\widetilde{\chi}_{1}^{0} \widetilde{\chi}_{1}^{0}$ production with decays via two $\mathrm{H}$ bosons. Each of the $\mathrm{H}$ bosons is expected to decay via $b \bar{b}$, hence, at least three $\mathrm{b}$-tagged jets and significant $p_{\mathrm{T}}^{\text {miss }}$ is required. The irreducible background is t $\overline{\mathrm{t}}$-like which contains two real b's in the final state and at least one additional light-flavor jet that is mistagged. A deep neural network (DNN) approach has been chosen, which improves the b-tagging efficiency by $20 \%$ with respect to conventional algorithms. The four jets with the highest b-tagging score in the event are used to reconstruct two $\mathrm{H}$ boson candidates such that the mass difference between the two is minimized. The average $\mathrm{H}$ boson candidate mass then is used for signal extraction. From the absence of any significant excess over the SM prediction, the exclusion mass range for the higgsino $\widetilde{\chi}_{1}^{0}$ reaches from 230 to $770 \mathrm{GeV}$.

\section{Conclusion}

In summary, the excellent performance of the LHC and the CMS detector in 2016 has provided enough data to probe new physics in yet unexplored territory. In the extensive search program conducted by CMS we benefit from improved analysis tools to search in the very difficult compressed regions of parameter space and by means of a boson that has been found only five years ago [12]. Interesting times are ahead of us as even better tools and even more data will help CMS to improve its sensitivity to these challenging topologies in the coming months and years. 


\section{References}

[1] CMS Collaboration, “The CMS Experiment at the CERN LHC”, JINST 3 (2008) S08004, doi:10.1088/1748-0221/3/08/S08004.

[2] C. Balázs, M. Carena, and C. E. M. Wagner, "Dark matter, light stops and electroweak baryogenesis", Phys. Rev. D 70 (2004) 015007, doi:10.1103/PhysRevD. 70.015007 , arXiv:hep-ph/0403224.

[3] CMS Collaboration, "Search for direct production of bottom and top squark pairs in proton-proton collisions at $\sqrt{s}=13 \mathrm{TeV}$ ", submitted to PLB, CMS-PAS-SUS-16-032.

[4] CMS Collaboration, "Search for new phenomena with the $M_{\mathrm{T} 2}$ variable in the all-hadronic final state produced in proton-proton collisions at $\sqrt{s}=13 \mathrm{TeV}$ ", submitted to EPJC, CMS-PAS-SUS-16-036.

[5] CMS Collaboration, "Search for direct production of supersymmetric partners of the top quark in the all-jets final state in proton-proton collisions at $\sqrt{s}=13 \mathrm{TeV}$ ", accepted by JHEP,

CMS-PAS-SUS-16-049.

[6] CMS Collaboration, "Search for supersymmetry in events with at least one soft lepton, low jet multiplicity, and missing transverse momentum in proton-proton collisions at $\sqrt{s}=13 \mathrm{TeV}$, CMS-PAS-SUS-16-052.

[7] CMS Collaboration, "Search for new physics in events with two low momentum opposite-sign leptons and missing transverse energy at $\sqrt{s}=13 \mathrm{TeV}$ ", CMS-PAS-SUS-16-048.

[8] CMS Collaboration, "Search for electroweak production of charginos and neutralinos in multilepton final states in proton-proton collisions at $\sqrt{s}=13 \mathrm{TeV}$ ", submitted to JHEP,

CMS-PAS-SUS-16-039.

[9] CMS Collaboration, "Search for electroweak production of charginos and neutralinos at $13 \mathrm{TeV}$ ", CMS-PAS-SUS-17-004.

[10] CMS Collaboration, "Search for electroweak production of charginos and neutralinos in WH events in proton-proton collisions at $\sqrt{s}=13 \mathrm{TeV}$ ", submitted to JHEP, CMS-PAS-SUS-16-043.

[11] CMS Collaboration, "Search for the pair production of Higgsinos in pp collisions at $\sqrt{s}=13 \mathrm{TeV}$ in final states with Higgs bosons and large missing transverse momentum”, submitted to PRD, CMS-PAS-SUS-16-044.

[12] CMS Collaboration, "Observation of a new boson at a mass of $125 \mathrm{GeV}$ with the CMS experiment at the LHC”, Phys. Lett. B 716 (2012) 30, doi : $0.1016 /$ j.physletb.2012.08.021, arXiv:1207.7235. 